\title{
Study on Multi-objective Flexible Job-shop Scheduling Problem considering Energy Consumption
}

\author{
Zengqiang Jiang, Le Zuo, Mingcheng E \\ School of Mechanical, Electronic \& Control Engineering, Beïing Jiaotong University (China) \\ zgijang@,bjtu.edu.cn,12121307@,bjtu.edu.cn,emch@,bjtu.edu.cn
}

Received: January 2014

Accepted: May 2014

\section{Abstract:}

Purpose: Build a multi-objective Flexible Job-shop Scheduling Problem(FJSP) optimization model, in which the makespan, processing cost, energy consumption and cost-weighted processing quality are considered, then Design a Modified Non-dominated Sorting Genetic Algorithm (NSGA-II) based on blood variation for above scheduling model.

Design/methodology/approach: A multi-objective optimization theory based on Pareto optimal method is used in carrying out the optimization model. NSGA-II is used to solve the model.

Findings: By analyzing the research status and insufficiency of multi-objective FJSP, Find that the difference in scheduling will also have an effect on energy consumption in machining process and environmental emissions. Therefore, job-shop scheduling requires not only guaranteeing the processing quality, time and cost, but also optimizing operation plan of machines and minimizing energy consumption.

Originality/value: A multi-objective FJSP optimization model is put forward, in which the makespan, processing cost, energy consumption and cost-weighted processing quality are considered. According to above model, Blood-Variation-based NSGA-II (BVNSGA-II) is designed. In which, the chromosome mutation rate is determined after calculating the blood relationship between two cross chromosomes, crossover and mutation strategy of NSGA-II is 
optimized and the prematurity of population is overcome. Finally, the performance of the proposed model and algorithm is evaluated through a case study, and the results proved the efficiency and feasibility of the proposed model and algorithm.

Keywords: multi-objective scheduling, flexible job-shop scheduling, NSGA-II, energy consumption, blood variation

\section{Introduction}

With the continuous development of productivity, on the one hand, human life is improving constantly, but on the other hand, which leads to tremendous damages to natural environment. So it's important to minimize the destruction to natural environment when making efforts to improve production efficiency. Energy consumption is one of important aspects. In actual production processes, scheduling is one of the key factors that influence production efficiency, quality and cost (Zhang, Dong, Wang, Li \& Liu, 2010). In addition, the difference in scheduling will also have an effect on resource consumption and emissions (He, Liu, Cao \& Liu, 2007). Therefore, job-shop scheduling requires not only guaranteeing the processing quality, time and cost, but also optimizing operation plan of machines to minimize energy consumption (Fang, Uhana, Zhao \& Sutherland, 2011; Luo, Du, Huang, Chen \& Li, 2013; Dai, Tang, Giret, Salido \& Li, 2013).

FJSP is an extension to traditional Job-Shop Scheduling Problem (Liu, Yang, Cheng, Xing, Lu, Zhao et al., 2012). It usually has multiple optimization objectives. S o a multi-objective optimization result is often not a single optimal solution, but a set of Pareto optimal solutions (Zheng, 2010).

Multi-objective optimization model generally adopts a method of single objective transformation, random weighting and optimization method based on Pareto (Xing, Chen \& Yang, 2009; Zhang, Shao, Li \& Gao, 2009; Xu, Ying \& Wang, 2010). A Pareto optimal method can obtain a set of Pareto optimal solutions in an optimizing process, which is consistent with an actual scheduling problem, so this method is favored by researchers ( $\mathrm{Li}$, Pan \& Wang, 2010; Ghasem \& Mehdi, 2011). Representative algorithms include the MOGA (Fonseca \& Fleming, 1993), NPGA (Horn, Nafpliotis \& Goldberg, 1994), NSGA and NSGA-II (Srinivas \& Deb, 1995; Deb, Pratap, Agarwal \& Meyarivan, 2002), PAES (Knowles \& Corne, 1999), SPEA and SPEA2 (Zitzler \& Thile, 1999), PESA and PESA-II (Corne, 2000), OMOEA (Zeng, Li, Ding \& Yao, 2004) and so on. Among them, NSGA-II is widely used because of its better distribution and faster convergence speed, but it also has a weakness of high computational complexity and premature. 
In this paper, taking into account the energy consumption and according to an actual production environment, a multi-objective optimization scheduling model is developed. In this model, energy consumption, makespan, processing cost and cost-weighted processing quality are optimized; and an NSGA-II with blood variation is designed by optimizing crossover and mutation strategy of NSGA-II in order to overcome the prematurity of population.

\section{Problem Description}

A multi-objective Flexible Job-Shop Scheduling Problem can be described as following:

Suppose that there is $m$ machines and $n$ workpieces, each work consists of several operations; workpiece $i$ includes $q_{i}$ procedures; each procedure can be processed by several machines; $a_{i j}{ }^{k}$ represents that procedure $j$ of workpiece $i$ is processed on machine $\mathrm{k}$ or not and it's value is 1 or 0 ; the processing cost, time, quality is relying on the performance of the machine; $S_{i j}^{k}$ is beginning time of procedure $j$ of processing workpiece $i$ on machine $k_{i} T_{i j}^{k}$

is the duration of procedure $j$ of processing workpiece $\mathrm{i}$ on machine $\mathrm{k}_{i} F_{i j}{ }^{k}$ is finishing time of procedure $j$ of processing workpiece $i$ on machine $k, F_{i j}{ }^{k}=S_{i j}{ }^{k}+T_{i j}{ }^{k}$. The scheduling goal is to determine processing sequence and processing machines, and to make each scheduling objective achieve optimal when satisfying the below constraints (Liu, Zhang, Jiang, Ge \& Zhang, 2008):

(1) Each machine can only process one workpiece at a time;

(2) All the machines are available at beginning time;

(3) Processing can't be interrupted;

(4) A processing plan has been determined, and all workpieces have the same priority;

(5) procedure $j$ can be started only after the finish of procedure $j-1$ for a workpiece.

\section{Scheduling Model}

In the scheduling model, the following four scheduling objective variables are included, which are cost, time, quality and energy consumption, the optimization objective of scheduling model can be written as: $\min (T, C, Q, E)$. $T$ represents the total processing time, $C$ is cost, $Q$ is quality and $E$ is energy consumption. 
1) Time

The processing time can be represented as make-span: $T=\max \left(F_{i j}{ }^{k}\right)=\max \left(S_{i j}{ }^{k}+T_{i j}{ }^{k}\right)$

2) Cost

The processing cost includes the cost of materials and production; the production cost is directly related to production scheduling (Liu et al., 2008).

Cost of raw materials:

$M C=\sum_{i=1}^{n} m c_{i}$, where $m c_{i}$ denotes the cost of raw materials of work $i$.

Process cost:

$P C=\sum_{i=1}^{n} \sum_{j=1}^{q_{i}} O_{i j}^{k} T_{i j}^{k} p c_{k}$, where $p c_{k}$ stands for the process cost of machine $k$ per unit time, in this cost the personnel cost is included.

Therefore, the final processing cost is $C=M C+P C=\sum_{i=1}^{n} m c_{i}+\sum_{i=1}^{n} \sum_{j=1}^{q_{i}} O_{i j}^{k} T_{i j}^{k} p c_{k}$.

3) Quality

Defective rate can be used to measure the processing quality of a procedure on an machine. Besides, as more and more processes of a workpiece have been done, the associated cost increases. If processing defects appear in the later stage of production processing, the loss of cost will be higher. So, the cost-weighted processing quality instability index $L_{i j}{ }^{k}$ is used to represent processing quality.

$P C_{i}^{H}=\sum_{j=1}^{H} O_{i j}^{k} T_{i j}^{k} p c_{k}$, where the process cost of first $H$ steps of the workpiece $i$.

$L_{i j}^{k}=\left(m c_{i}+P C_{i}^{j}\right) q_{i j}^{k}, q_{i j}{ }^{k}$ is the defective rate of procedure $j$ of workpiece $i$ on machine $k$. $m c_{i}+P C_{i}^{j}$ is the cost-weighted quality instability index, indicating that when a workpiece costs more, its quality is more important.

$Q=\sum_{i=1}^{n} \sum_{j=1}^{q_{i}} O_{i j}^{k} L_{i j}^{k} \quad$ calculates the cost-weighted processing quality instability index of scheduling. 
4) Energy Consumption

The energy consumption to complete the same process on different machines is different. Therefore, energy consumption should be represented as total energy consumption $E$, $E=\sum_{i=1}^{n} \sum_{j=1}^{q_{i}} O_{i j}^{k} T_{i j}^{k} e_{k}$

\section{Scheduling algorithm of BVNSGA-II}

NSGA-II has a better rate of convergence, so it is widely used. But it lacks population diversity. A new NSGA-II algorithm is developed, which is named Blood-Variation-based NSGA-II(BVNSGA-II) and its crossover and mutation strategy is improved. In BVNSGA-II, the mutation rate is determined by calculating chromosome blood relationship. This new algorithm can avoid early convergence to local optimal solution.

\subsection{Encoding and decoding}

FJSP should get the sequence of a procedure and the proper machine for every procedure.

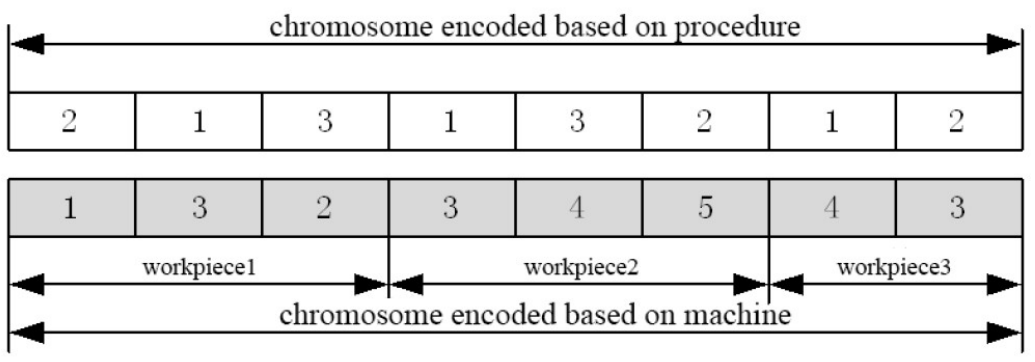

Figure 1. 2-level integer encoding based on procedure and machine

As shown in Figure1, the encoding consists of two parts. The first part is encoding based on process, which can determine the sequence of working procedure. The other part is encoding based on machine, which can choose the machine for each procedure. Therefore, a chromosome in Figure 2 shows 3 workpieces, which consists of 8 procedures, and will be processed on 5 different machines. The processing sequence of this chromosome can be represented as $O_{21}^{3}, O_{11}^{1}, O_{31}^{4}, O_{12}^{3}, O_{32}^{3}, O_{22}^{4}, O_{13}^{2}, O_{23}^{5}$ represents that the $j^{\text {th }}$ procedure of the $i$ th workpiece will be processed on the $k^{\text {th }}$ machine. For example, $O_{21}^{3}$ means that the first procedure of the workpiece 2 will be processed on the machine 3 . 


\subsection{Select operation}

Compared to a single objective optimization problem, the select operation of a multi-objective optimization problem is more complex, which generally contains the ranking of individuals and selection strategy of non-dominated solutions with the same rank.

\subsubsection{Ranking of individuals}

At present, two kinds of Pareto sorting methods are widely used: the recursion sorting method (Jensen, 2003) and the modified quick sorting method (Zheng, 2010). Research shows that the second method has better computational performance when there are only two objectives (Zheng, 2010). So in this paper, the modified quick sorting algorithm will be used.

\subsubsection{Selection strategy of non-dominated solution with the same rank}

The individuals will have different ranks after non-dominated sorting, and individuals with low rank will be chosen to participate in the evolution. When the choosing individuals with the same rank, some strategies should be taken to make sure the diversity of colony in the evolution. At present some strategies are commonly used, which include niche technology, information entropy, density based clustering, grid, and classification etc. In this paper, the strategy of density based clustering is adopted. Although it has slightly higher computational complexity, it can capture the diversity and distribution of population macroscopically, and can also characterize the relationship among individuals with capability of controlling the colony in the evolution process (Zheng, 2010). Set $P[i]_{\text {dis }}$ as the crowding distance of individual $i$, and $P[i]_{\mathrm{k}}$ as the function value of individual $i$ in subgoal $k$. So in normal circumstance, when there are $r$ subgoals, the crowding distance of individual $i$ will be

$$
P[i]_{d i s}=\sum_{k=1}^{r}\left(P[i+1]^{k}-P[i-1]^{k}\right)
$$

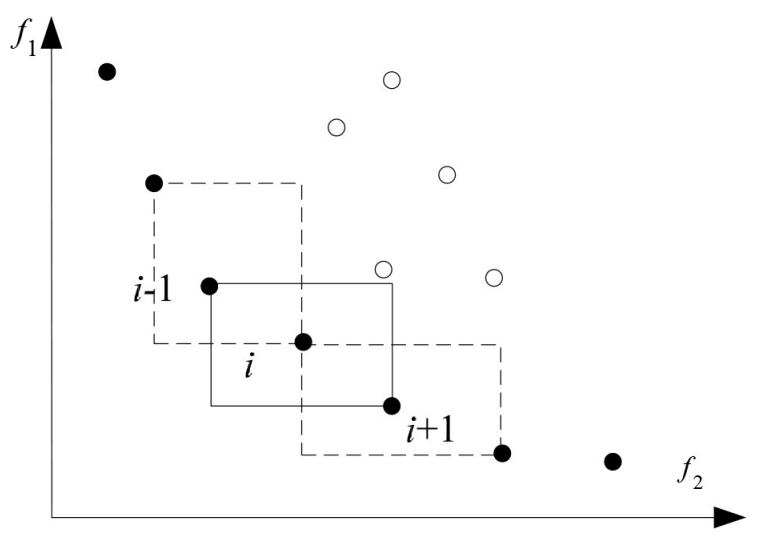

Figure 2. the crowding distance of two neighbouring individuals 
As shown in Figure 2, if there are only 2 subgoals, the crowding distance of individual $i$ is the sum of the length and width of the Solid rectangle in Figure 2.

In order to maintain the diversity of population, the individuals with larger crowding distances have higher probability to take part in reproduction and evolution.

\subsection{Crossover and Mutation Operation}

BVNSGA-II improves the crossover and mutation strategy of NSGA-II. It calculates the blood relationship of two chromosomes before genes crossover. Then, according to the calculation, it computes mutation rates of new chromosomes. At last, it will perform mutation operation to the crossed genes according to the mutation rate, which can avoid the prematurity of the algorithm.

\subsubsection{Calculation method of genetic relative index and mutation probability}

A consanguineous crossover will easily result in premature convergence of the algorithm, so according to different blood relationship of child chromosomes, different mutation rates to be used to operate mutation. Assume that the gene set of two chromosomes to be crossed is $P_{1}$ and $P_{2}, s$ is consanguineous relative index and $v$ is the variation index, and $V$ is the initial mutation rate. $s$ and $v$ is calculated by the follow program.

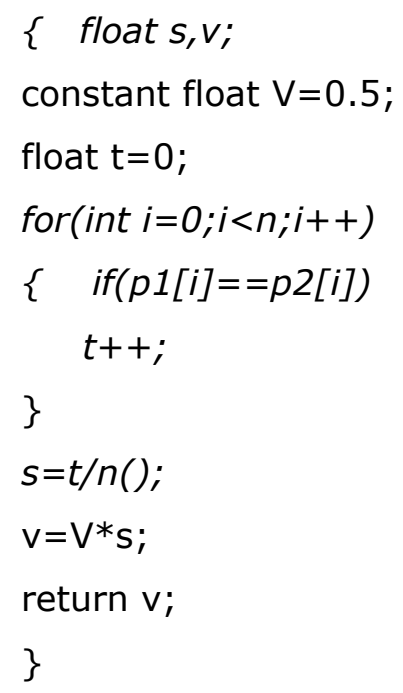




\subsubsection{Crossover Strategy of Processing Sequence}

The cross process of processing sequence gene segments is shown as Figure 3. $P_{1}$ and $P_{2}$ are two parent chromosomes, $P_{1}{ }^{\prime}$ and $P_{2}{ }^{\prime}$ are the progenies after crossover. The steps of crossover are following.

Step 1: Divide the workpieces into two sets, $J_{1}$ and $J_{2}$, and assume that the result is $J_{1}=\{1,3\}$, $J_{2}=\{2\}$.

Step 2: Copy the workpieces included in the $J_{1}$ and $P_{1}$ to $P_{1}^{\prime}$; copy the workpieces included in the $J_{2}$ and $P_{2}$ to $P_{2}^{\prime} ;$ do not change the gene location of these workpieces at the same time.

Step 3: Copy the workpieces included in the $J_{2}$ and $P_{2}$ to $P_{1}^{\prime}$; copy the workpieces included in the $J_{1}$ and in $P_{1}$ to $P_{2}^{\prime}$; do not change the gene sequence of these workpieces at the same time.

\begin{tabular}{|c|c|c|c|c|c|c|c|c|}
\hline$P_{1}^{\prime}$ & 1 & 2 & 3 & 1 & 3 & 2 & 1 & 2 \\
\hline & $\uparrow$ & & $\uparrow$ & $\uparrow$ & $\uparrow$ & & $\uparrow$ & \\
\hline$P_{1}$ & $1_{1}$ & 2 & 3 & 11 & $3^{\prime}$ & 2 & 1 & 2 \\
\hline$P_{2}$ & 11 & 3 & $2)$ & 1 & 2 & 1 & 2 & 3 \\
\hline & $\downarrow$ & $\nabla$ & $\downarrow$ & $\downarrow$ & $\downarrow$ & 4 & $\downarrow$ & 1 \\
\hline$P_{2}^{\prime}$ & 1 & 3 & $\frac{1}{2}$ & 1 & 2 & 3 & 2 & 1 \\
\hline
\end{tabular}

Figure 3. Crossover of processing sequence

\subsubsection{Crossover Strategy of Machine Allocation}

Figure 4 shows the crossover operation of machine allocation. Assume that $P_{1}$ and P2 cross and get $P_{1}^{\prime}$ and $P_{2}{ }^{\prime}$. The specific steps of crossover are as follow.

Step 1: Make a set $R$, which consists of 0 and 1, with the same length of a chromosome. Assume that $R=\{0,1,0,1,1,0,1,0\}$.

Step 2: Select the procedures whose location are the same to element 1 in $\mathrm{R}$ from $P_{1}$ and $P_{2}$. As shown in the grey part in Figure 4.

Step 3: Cross the machine genes in grey part, and keep other parts to its child, then get child chromosomes $P_{1}^{\prime}$ and $P_{2}{ }^{\prime}$. 


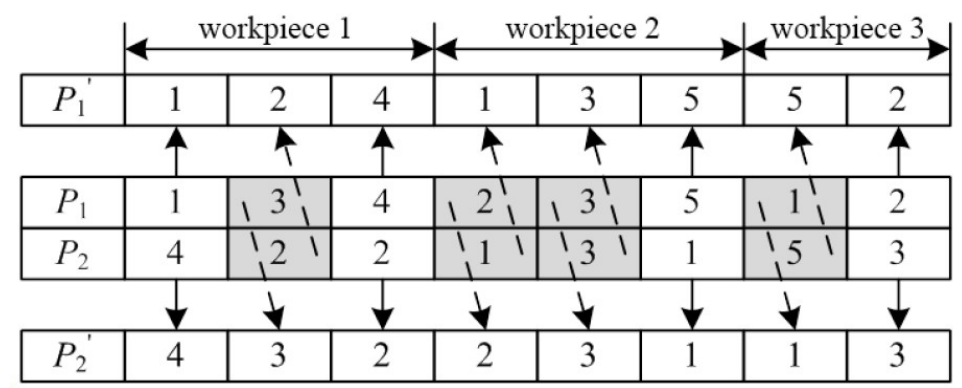

Figure 4. Crossover strategy of machine allocation

\subsubsection{Mutation Operation}

The mutation operation used in this paper consists of two parts.

1) The mutation operation of gene segments of processing sequence: as shown in Figure 5, firstly choose a processing gene from one workpiece (assuming it is the grey 2 in Figure5), and then insert it into a random location.

\begin{tabular}{|l|l|l|l|l|l|l|l|l|}
\hline$P_{1}^{\prime}$ & 1 & 2 & 3 & 2 & 1 & 3 & 1 & 2 \\
\hline$P_{1}$ & 1 & 2 & 3 & 1 & 3 & 2 & 1 & 2 \\
\hline
\end{tabular}

Figure 5. Mutation operation of processing sequence

2) The mutation operation of gene segments of machine allocation: as shown in Figure 6, firstly choose a machine gene randomly in machine gene segments, and then choose the other machines in machine set that apply to this gene to replace the old one.

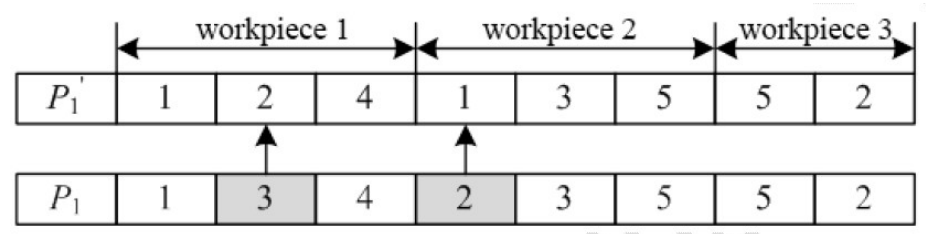

Figure 6. Mutation operation based on machine encoding

\subsection{Algorithm Flowchart}

The algorithm flowchart of BVNSGA-II is shown in Figure 7. Firstly, choose randomly some pairs of individuals from the parent population $P_{t}$, and calculate the blood relationship of this pair that will crossover, and then calculate the mutation rate of the child individuals get from 
crossover. After that, mutate these individuals according their mutation rates, and get the child population $Q_{t}$. Combine the parent population $P_{t}$ and the child population $Q_{t}$. Compute the individual rank in population by non-dominated sorting and the calculation of crowding distance, and trim the population to a new population $P_{t+1}$ according to the ranking. Repeat the above operations until the termination condition of the algorithm is achieved.

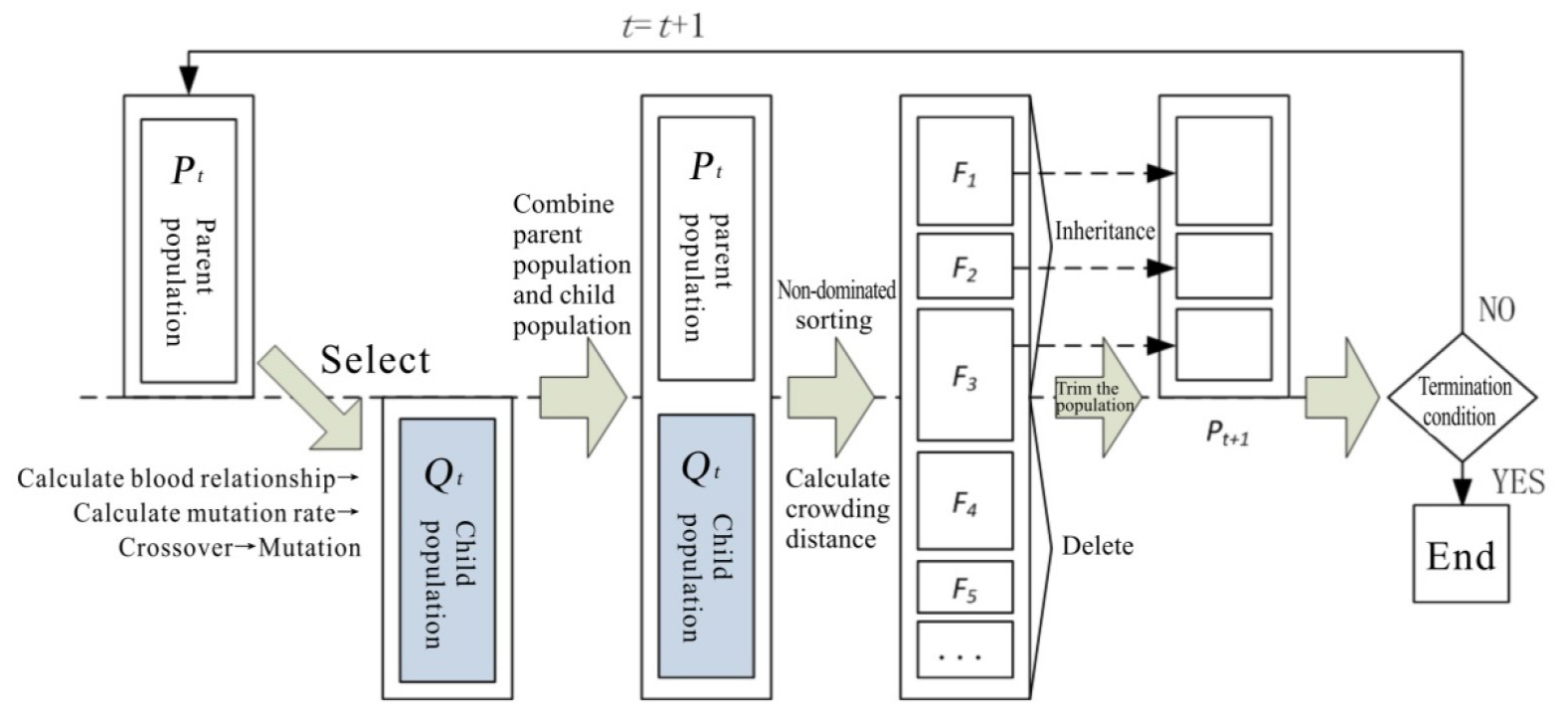

Figure 7. Process of NSGA-II based on blood variation

\section{Case Study}

Table 1 shows these data of a FJSP instance of mechanical workshop: the arrival time of the workpieces, the processing time of each procedure, the processing quality, the cost of raw materials, the processing cost of machine in unit time and energy consumption of machine in unit time. The relevant parameters of the algorithm are: the population size is 50 , evolution generation is 100 , crossover rate is $100 \%$, and initial mutation rate is $10 \%$.

Because the multi-objective optimization problem in this paper has 4 objectives to optimize, it cannot be shown in the Pareto hyper surface by an intuitive graph. Table 2 shows the final set of Pareto solutions with 50 individuals. 


\begin{tabular}{|c|c|c|c|c|c|c|c|c|c|}
\hline \multirow[b]{2}{*}{$\boldsymbol{J}^{i}$} & \multirow[b]{2}{*}{$o^{i j}$} & \multirow[b]{2}{*}{$\boldsymbol{R}^{i}$} & \multicolumn{6}{|c|}{$T_{i j}^{k} / q_{i j}^{k}$} & \multirow{2}{*}{$m c^{i}$} \\
\hline & & & $M^{1}$ & $M^{2}$ & $M^{3}$ & $M^{4}$ & $M^{5}$ & $M_{6}$ & \\
\hline \multirow{6}{*}{$J^{1}$} & $O^{11}$ & \multirow{6}{*}{6} & $12 / 0.10$ & $18 / 0.20$ & - & $18 / 0.05$ & - & $16 / 0.15$ & \multirow{6}{*}{120} \\
\hline & $O^{12}$ & & - & $8 / 0.1$ & $6 / 0.11$ & $20 / 0.06$ & $20 / 0.04$ & & \\
\hline & $O^{13}$ & & - & $8 / 0.15$ & - & - & $18 / 0.1$ & $10 / 0.2$ & \\
\hline & $O^{14}$ & & $14 / 0.16$ & - & $16 / 0.08$ & $16 / 0.1$ & - & - & \\
\hline & $O^{15}$ & & $8 / 0.18$ & $8 / 0.2$ & $10 / 0.12$ & $10 / 0.15$ & $6 / 0.22$ & $12 / 0.02$ & \\
\hline & $0^{16}$ & & - & - & $20 / 0.1$ & - & $16 / 0.2$ & $18 / 0.15$ & \\
\hline \multirow{3}{*}{$J^{2}$} & $O^{21}$ & \multirow{3}{*}{2} & $18 / 0.20$ & - & $8 / 0.10$ & $20 / 0.06$ & $14 / 0.12$ & - & \multirow{3}{*}{100} \\
\hline & $O^{22}$ & & - & $18 / 0.08$ & $12 / 0.09$ & $9 / 0.11$ & - & $15 / 0.06$ & \\
\hline & $O^{23}$ & & $7 / 0.12$ & - & $20 / 0.11$ & $12 / 0.15$ & $18 / 0.2$ & - & \\
\hline \multirow{5}{*}{$J^{3}$} & $O^{31}$ & \multirow{5}{*}{2} & $18 / 0.13$ & $20 / 0.15$ & $8 / 0.2$ & $7 / 0.06$ & $6 / 0.10$ & - & \multirow{5}{*}{65} \\
\hline & $0^{32}$ & & - & $7 / 0.11$ & $8 / 0.12$ & - & $20 / 0.20$ & - & \\
\hline & $0^{33}$ & & $7 / 0.23$ & $10 / 0.12$ & - & $14 / 0.07$ & - & $20 / 0.02$ & \\
\hline & $0^{34}$ & & - & $8 / 0.15$ & $20 / 0.01$ & $16 / 0.1$ & $15 / 0.11$ & - & \\
\hline & $0^{35}$ & & $20 / 0.05$ & - & $18 / 0.15$ & $10 / 0.1$ & $10 / 0.2$ & $15 / 0.13$ & \\
\hline \multirow{5}{*}{$J_{4}$} & $0^{41}$ & \multirow{5}{*}{5} & $22 / 0.01$ & - & $19 / 0.1$ & $16 / 0.11$ & $20 / 0.1$ & - & \multirow{5}{*}{100} \\
\hline & $0^{42}$ & & - & $13 / 0.21$ & $10 / 0.2$ & $18 / 0.15$ & $10 / 0.17$ & $18 / 0.2$ & \\
\hline & $0^{43}$ & & $6 / 0.25$ & $10 / 0.13$ & - & - & - & $20 / 0.01$ & \\
\hline & $O^{44}$ & & $12 / 0.12$ & - & $8 / 0.18$ & - & $8 / 0.1$ & - & \\
\hline & $O^{45}$ & & $20 / 0.02$ & $12 / 0.15$ & $20 / 0.1$ & $15 / 0.18$ & - & - & \\
\hline \multirow{6}{*}{$J_{5}$} & $O^{51}$ & \multirow{6}{*}{10} & - & $8 / 0.20$ & - & $10 / 0.15$ & $15 / 0.11$ & $10 / 0.1$ & \multirow{6}{*}{150} \\
\hline & $0^{52}$ & & $12 / 0.2$ & - & $15 / 0.15$ & $20 / 0.08$ & - & $8 / 0.24$ & \\
\hline & $O^{53}$ & & $16 / 0.12$ & $15 / 0.12$ & - & - & $20 / 0.08$ & $10 / 0.21$ & \\
\hline & $O^{54}$ & & - & $6 / 0.25$ & $8 / 0.15$ & $8 / 0.2$ & $15 / 0.04$ & - & \\
\hline & $O^{55}$ & & $15 / 0.08$ & - & - & $10 / 0.09$ & - & $12 / 0.08$ & \\
\hline & $O^{56}$ & & $13 / 0.06$ & $5 / 0.26$ & $13 / 0.1$ & $15 / 0.08$ & $20 / 0.01$ & $7 / 0.2$ & \\
\hline \multirow{4}{*}{$J_{6}$} & $O^{61}$ & \multirow{4}{*}{4} & - & $14 / 0.2$ & - & - & $9 / 0.2$ & $10 / 0.22$ & \multirow{4}{*}{85} \\
\hline & $0^{62}$ & & - & - & $12 / 0.17$ & $15 / 0.15$ & - & $9 / 0.2$ & \\
\hline & $0^{63}$ & & $13 / 0.03$ & $7 / 0.1$ & - & $16 / 0.02$ & $9 / 0.17$ & - & \\
\hline & $0^{64}$ & & $20 / 0.01$ & - & $13 / 0.09$ & - & - & $17 / 0.06$ & \\
\hline \multicolumn{3}{|c|}{$p c_{k}$} & 6 & 8 & 7 & 4 & 5 & 6 & \\
\hline \multicolumn{3}{|c|}{$e_{k}$} & 8 & 10 & 7.5 & 12 & 9 & 10.5 & \\
\hline
\end{tabular}

Table 1. Instance data 


\begin{tabular}{|c|c|c|c|c|}
\hline Number & $\begin{array}{c}\text { Makespan } \\
\text { (T) }\end{array}$ & $\begin{array}{l}\text { Processing } \\
\text { cost (C) }\end{array}$ & $\begin{array}{l}\text { Processing } \\
\text { quality (Q) }\end{array}$ & $\begin{array}{c}\text { Energy } \\
\text { consumption } \\
\text { (E) }\end{array}$ \\
\hline 1 & 86 & 2189 & 1213.06 & 2653 \\
\hline 2 & 100 & 2229 & 1270.98 & 2548 \\
\hline 3 & 206 & 2472 & 775.3 & 3324.5 \\
\hline 4 & 92 & 2270 & 1208.51 & 2511 \\
\hline 5 & 133 & 2120 & 1151.41 & 2812.5 \\
\hline 6 & 218 & 2443 & 792.53 & 3382 \\
\hline 7 & 227 & 2612 & 697.1 & 3701.5 \\
\hline 8 & 107 & 2198 & 1261.83 & 2574 \\
\hline 9 & 236 & 2568 & 741.42 & 3499.5 \\
\hline 10 & 117 & 2339 & 1066.89 & 2759 \\
\hline 11 & 141 & 2172 & 1141.99 & 2718.5 \\
\hline 12 & 188 & 2427 & 822.1 & 3374 \\
\hline 13 & 220 & 2640 & 700.51 & 3650.5 \\
\hline 14 & 118 & 2435 & 942.06 & 3038.5 \\
\hline 15 & 147 & 2288 & 1040.7 & 2944 \\
\hline 16 & 137 & 2472 & 847.67 & 3345.5 \\
\hline 17 & 112 & 2243 & 1098.5 & 2725 \\
\hline 18 & 160 & 2291 & 1022.07 & 3187.5 \\
\hline 19 & 105 & 2406 & 979.06 & 3000 \\
\hline 20 & 144 & 2510 & 873.71 & 3180.5 \\
\hline 21 & 148 & 2572 & 839.45 & 3483 \\
\hline 22 & 192 & 2496 & 762.21 & 3511.5 \\
\hline 23 & 108 & 2254 & 1221.82 & 2575 \\
\hline 24 & 100 & 2218 & 1284.92 & 2559 \\
\hline 25 & 101 & 2244 & 1153.01 & 2677.5 \\
\hline 26 & 227 & 2612 & 697.1 & 3701.5 \\
\hline 27 & 167 & 2404 & 945.73 & 2998.5 \\
\hline 28 & 220 & 2654 & 672.47 & 3762.5 \\
\hline 29 & 145 & 2672 & 718.76 & 3459.5 \\
\hline 30 & 129 & 2461 & 903.55 & 3312.5 \\
\hline 31 & 112 & 2207 & 1067.31 & 2905.5 \\
\hline 32 & 149 & 2440 & 809.02 & 3300.5 \\
\hline 33 & 200 & 2532 & 706.65 & 3625.5 \\
\hline 34 & 89 & 2291 & 1186.55 & 2713 \\
\hline 35 & 210 & 2614 & 707.09 & 3568.5 \\
\hline 36 & 169 & 2348 & 982.7 & 2924 \\
\hline 37 & 143 & 2555 & 879.46 & 3220 \\
\hline 38 & 193 & 2652 & 685.43 & 3776.5 \\
\hline 39 & 172 & 2498 & 758.93 & 3507 \\
\hline 40 & 178 & 2383 & 860.77 & 3402 \\
\hline 41 & 150 & 2359 & 930.47 & 3097.5 \\
\hline 42 & 127 & 2318 & 1056.72 & 2863 \\
\hline 43 & 206 & 2472 & 775.3 & 3324.5 \\
\hline 44 & 177 & 2599 & 793.2 & 3460 \\
\hline 45 & 134 & 2216 & 1114.28 & 2791.5 \\
\hline 46 & 236 & 2568 & 741.42 & 3499.5 \\
\hline 47 & 161 & 2341 & 992.78 & 2847.5 \\
\hline 48 & 99 & 2196 & 1163.29 & 2613.5 \\
\hline 49 & 119 & 2448 & 964.36 & 2973.5 \\
\hline 50 & 154 & 2395 & 966.8 & 3066.5 \\
\hline
\end{tabular}

Table 2. The Pareto optimal set of the final generation 
The final result is a set of Pareto optimal solution which includes lots of individuals, and it provides a series of feasible optimal solutions. In production processing, decision makers can determine the weights of different objectives by AHP (Analytic Hierarchy Process) according to the actual production situation. Assume the weights of $T, C, Q$ and $E$ are $0.5,0.3,0.1$ and 0.1 . then calculate the different $O b j_{i}$ of each optimal solution in the set of the Pareto optimal solution by using the following formula.

$$
\mathrm{Obj}_{i}=w_{T} \frac{T_{\max }-T_{i}}{T_{\max }-T_{\min }}+w_{C} \frac{C_{\text {max }}-C_{i}}{C_{\max }-C_{\min }}+w_{Q} \frac{Q_{\max }-Q_{i}}{Q_{\max }-Q_{\min }}+w_{E} \frac{E_{\max }-E_{i}}{E_{\max }-E_{\min }}
$$

Sort the results and the $29^{\text {th }}$ solution is optimal, its Gantt-chart is shown in figure 8.

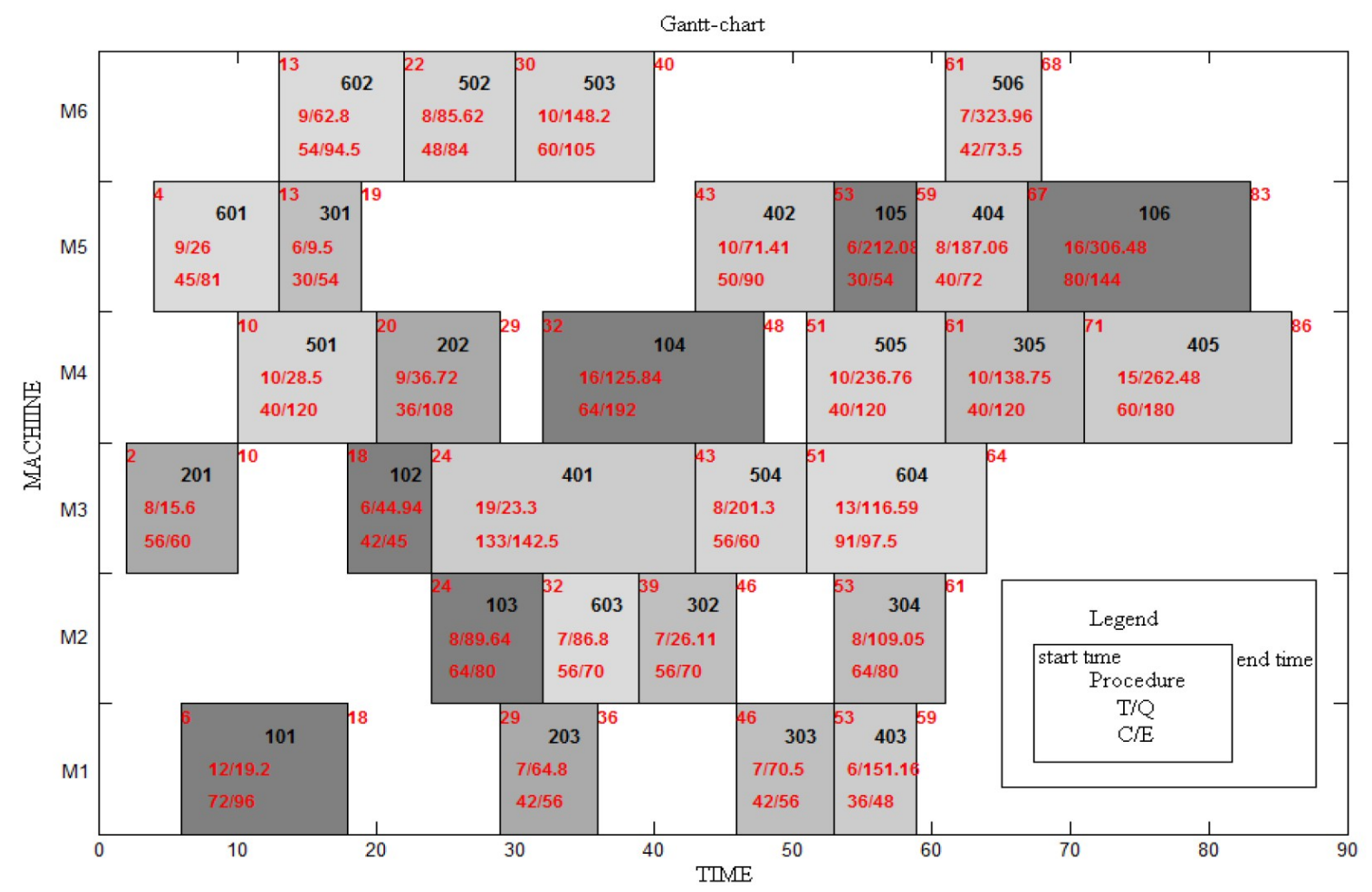

Figure 8. Gantt chart of The optimal solution

\section{Conclusion}

In this paper, a scheduling model is developed, which has 4 optimization objectives, quality, time, cost and energy consumption, and in which the energy consumption of machines is considered, so the scheduling plan is optimal not only in time, cost and quality but also in energy consumption. 
Then a method based on BVNSGA-II is proposed, in which, the chromosome mutation rate is determined after calculating the blood relationship between the two cross chromosomes, crossover and mutation strategy of NSGA-II is optimized and the prematurity of population is overcome, so the calculating speed and quality of above model is improved.

Finally, the performance of the proposed model and algorithm is evaluated through a case study, and the result proved the efficiency and feasibility of the proposed.

Though we have achieved some positive results, there are still some shortcomings in our research that need to be improved. For example, the states of a processing machine can be divided into stop, start-up, no-load, processing, and different states have different energy consumption, so I will try to find out the energy consumption features in different states by experiments, then to build a more precise scheduling model and find a more appropriated scheduling plan.

\section{Acknowledgment}

This work is supported by the Fundamental Research Funds for the Central Universities of China (Grant No.2012JBM090).

\section{References}

Corne, D.W. (2000). The Pareto envelope based selection algorithm for multi-objective optimization. Lecture Notes in Computer Science, 839-848. http://dx.doi.org/10.1007/3-54045356-3_82

Dai, M., Tang, D.B., Giret, A., Salido, M.A. \& Li, W.D. (2013). Energy-efficient scheduling for a flexible flow shop using an improved genetic-simulated annealing algorithm. Robotics and Computer-Integrated Manufacturing. 29(5), 418-429. http://dx.doi.org/10.1016/j.rcim.2013.04.001

Deb, K., Pratap, A., Agarwal, S., \& Meyarivan, T. (2002). A fast and elitist multi-objective genetic algorithm: NSGA-II. IEEE Transactions on Evolutionary Computation, 6(2): 182-197. http://dx.doi.org/10.1109/4235.996017

Fang, K., Uhana, N., Zhao, F., \& Sutherland, J.W. (2011). A new approach to scheduling in manufacturing for power consumption and carbon footprint reduction. Journal of Manufacturing Systems, 30, 234-240. http://dx.doi.org/10.1016/j.jmsy.2011.08.004

Fonseca, C.M., \& Fleming, P.J. (1993). Genetic algorithms for multiobjective optimization: Formulation, discussion and generalization. Proceedings of the Fifth International Conference on Genetic Algorithms, 416-423. 
Ghasem, M., \& Mehdi, M. (2011). A Pareto approach to multi-objective flexible job-shop scheduling problem using particle swarm optimization and local search. International Journal of Production Economics, 129(1), 14-22. http://dx.doi.org/10.1016/j.ijpe.2010.08.004

He, Y., Liu, F., Cao, H.J., \& Liu, C. (2007). Job Scheduling Model of Machining System for Green Manufacturing. Journal of Mechanical Engineering, 43(4), 27-33.

http://dx.doi.org/10.3901/JME.2007.04.027

Horn, J., Nafpliotis, N., \& Goldberg, D.E. (1994). A niched Pareto genetic algorithm for multi-objective optimization. Proceedings of the 1st IEEE Congress on Evolutionary Computation. Piscataway: IEEE, 82-87. http://dx.doi.org/10.1109/ICEC.1994.350037

Jensen, M.T. (2003). Reducing the run-time complexity of multiobjective EAs: The NSGA-II and other algorithms. IEEE Transactions on Evolutionary Computation, 7(5), 503-515. http://dx.doi.org/10.1109/TEVC.2003.817234

Knowles, J., \& Corne, D. (1999). The Pareto archived evolution strategy: a new baseline algorithm for multi-objective optimization. Proceedings of the 1999 Congress on Evolutionary Computation. Piscataway, NJ: IEEE Press, 98-105. http://dx.doi.org/10.1109/CEC.1999.781913

Li, J.Q., Pan, Q.K., \& Wang, Y.T. (2010). Hybrid Pareto-Based Tabu Search Algorithm for Solving the Multi-Objective Flexible Job Shop Scheduling Problem. Computer Integrated Manufacturing Systems, 16(7), 1419-1426.

Liu, A.J., Yang, Y., Cheng, W.M., Xing, Q.S., Lu, H., Zhao, X.H. et al. (2012). Improved NSGA for Complex Manufacturing Environment. Computer Integrated Manufacturing Systems, 18(11), 2447-2458.

Liu, M.Z., Zhang, M.W., Jiang, Z.Q., Ge, M.G., \& Zhang, M.X. (2008). Multi-objective and Flexible Job-Shop Problems Study Based on Hybrid Particle Swarm Optimization. Transactions of The Chinese Society For Agricultural Machinery, 39(5), 122-127.

Luo, H., Du, B., Huang, G.Q., Chen, H. \& Li, X. (2013). Hybrid flow shop scheduling considering machine electricity consumption cost. International Journal of Production Economics. 146(2), 423-439. http://dx.doi.org/10.1016/j.ijpe.2013.01.028

Srinivas, N., \& Deb, K. (1995). Multi-objective function optimization using non-dominated sorting genetic algorithm. Evolutionary Computation, 2(3), 221-248.

http://dx.doi.org/10.1162/evco.1994.2.3.221

Xing, L.N., Chen, Y.W., \& Yang, K.W. (2009). Multi-objective flexible job shop schedule: design and evaluation by simulation modeling. Applied Soft Computing, 9(1), 362-376. http://dx.doi.org/10.1016/j.asoc.2008.04.013 
Xu, X.L., Ying, S.Y., Wang, W.L. (2010). Fuzzy Flexible Job-Shop Scheduling Method Based on Multi-Agent Immune Algorithm. Control and Decision, 25(2), 171-184. (in Chinese).

Zhang, C.Y., Dong, X., Wang, X.J., Li, X.Y., \& Liu, Q. (2010). Improved NSGA-II for the Multi-objective Flexible Job-shop Scheduling Problem. Journal of Mechanical Engineering, 46(11), 156-164. http://dx.doi.org/10.3901/JME.2010.11.156

Zhang, G.H., Shao, X.Y., Li, P.G., Gao, L. (2009). An effective hybrid particles warm optimization algorithm for multi-objective flexible job shop scheduling problem. Computers \& Industrial Engineering, 56(2), 1309-1318. http://dx.doi.org/10.1016/j.cie.2008.07.021

Zheng, J.H. (2010). Multi-objective Evolutionary Algorithm and Application. Beijing: Science press.

Zitzler, E., \& Thile, L. (1999). Multi-objective evolutionary algorithm: A comparative case study and the strength Pareto approach. IEEE Transactions on Evolutionary Computation, 3(4), 257-271. http://dx.doi.org/10.1109/4235.797969

Zeng, S.Y., Li, H., Ding, L.X., \& Yao, S.Z. (2004). A Fast Algorithm for Finding Non-Dominated Set Based on Sorting. Journal Of Computer Research And Development, 41(9), 1565-1571.

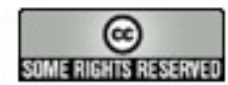

Article's contents are provided on a Attribution-Non Commercial 3.0 Creative commons license. Readers are allowed to copy, distribute and communicate article's contents, provided the author's and Journal of Industrial Engineering and Management's names are included. It must not be used for commercial purposes. To see the complete license contents, please visit http://creativecommons.org/licenses/by-nc/3.0/. 